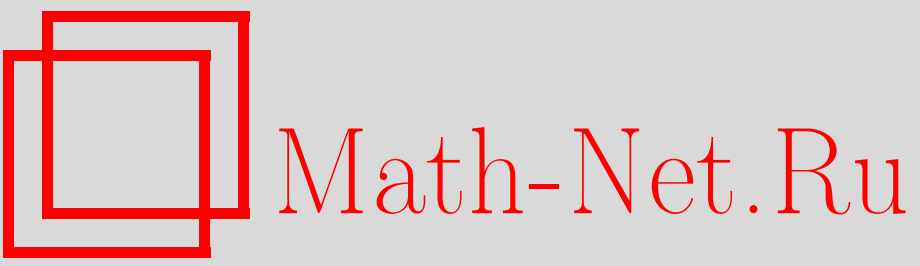

А. В. Цыганов, О построении переменных разделения для конечномерных интегрируемых систем, ТМФ, 2001, том 128, номер 2, 205-225

DOI: https://doi.org/10.4213/tmf492

Использование Общероссийского математического портала Math-Net.Ru подразумевает, что вы прочитали и согласны с пользовательским соглашением

http://www . mathnet.ru/rus/agreement

Параметры загрузки:

IP : 54.237 .59 .107

26 апреля 2023 г., $17: 37: 56$ 


\section{О ПОСТРОЕНИИ ПЕРЕМЕННЫХ РАЗДЕЛЕНИЯ ДЛЯ КОНЕЧНОМЕРНЫХ ИНТЕГРИРУЕМЫХ СИСТЕМ}

Рассматривается класс интегрируемых систем, для которых решения уравнения Гамильтона-Якоби зависят от $(n+m)$ произвольных параметров и представляются в виде произведения плоских кривых. Первые $n$ параметров отождествляются со значениями интегралов движения. Остающиеся параметры входят в определение интегралов движения как произвольные константы (заряды) и могут быть использованы для нахождения переменных разделения. Показано, что на орбитах коприсоединенного представления групп Ли операторы Казимира не только порождают семейство интегралов, но и позволяют построить переменные разделения.

\section{1. ОСНОВНЫЕ ОПРЕДЕЛЕНИЯ}

Пусть $(\mathcal{M}, \Omega)$ - симплектическое многообразие размерности $2 n$. Уравнение Гамильтона-Якоби

$$
\mathcal{Q}^{2 n-1}: \quad H-E=0, \quad E \in \mathbb{R},
$$

определяет гиперповерхность $\mathcal{Q}^{2 n-1}$ размерности $(2 n-1)$ в многообразии $\mathcal{M}$ [1], [2]. Произвольное $n$-мерное лагранжево многообразие $\mathcal{C}^{(n)} \in \mathcal{Q}^{2 n-1}$, является решением уравнения Гамильтона-Якоби. По теореме Лиувилля для интегрируемых систем соответствуюшее лагранжево многообразие зависит по крайней мере от $n$ параметров $\alpha_{1}, \ldots, \alpha_{n}$, которые отождествляются со значениями интегралов движения $I_{j}=$ $\alpha_{j}[1],[2]$.

В методе разделения переменных лагранжево многообразие ишется в виде произведения плоских кривых

$$
\mathcal{C}^{(n)}: \mathcal{C}_{1} \times \mathcal{C}_{2} \times \cdots \times \mathcal{C}_{n}
$$

Соответствующие кривые $\mathcal{C}_{j}$ на плоскости могут быть заданы либо параметрически, либо с помошью уравнений вида

$$
\mathcal{C}_{j}: \quad \Phi_{j}\left(\mu_{j}, \lambda_{j}, \alpha_{1}, \ldots, \alpha_{n}\right)=0, \quad j=1, \ldots, n, \quad \alpha_{j} \in \mathbb{R} .
$$

* Санкт-Петербургский государственный университет, Санкт-Петербург, Россия. E-mail: tsiganov@mph.phys.spbu.ru 
Здесь модули кривых $\alpha_{j}$ являются значениями заданного набора интегралов движения $I_{j}=\alpha_{j}$, a $\left(\mu_{j}, \lambda_{j}\right)$ - некоторые координаты на $j$-й плоскости, для которых в дальнейшем будем опускать индекс $j$.

Возможность представления лагранжева многообразия $\mathcal{C}^{(n)}$ в виде произведения плоских кривых не зависит от способа задания этих кривых (1.2) и выбора локальных координат фазового пространства. Однако об обшем инвариантном анализе данного геометрического свойства лагранжевых многообразий нам не известно.

Будем называть интегрируемую систему однородной, если соответствуюшее лагранжево многообразие

$$
\mathcal{C}^{(n)}: \overbrace{\mathcal{C} \times \cdots \times \mathcal{C}}^{n \text { раз }}
$$

представляется в виде произведения $n$ копий одной единственной плоской кривой $\mathcal{C}$.

Для некомпактных поверхностей уровня интегралов будем называть интегрируемую систему квазиоднородной, если соответствуюшее лагранжево многообразие

$$
\mathcal{C}^{(n)}: \overbrace{\mathcal{C} \times \cdots \times \mathcal{C}}^{(n-k) \text { раз }} \times \overbrace{\mathbb{C} \times \cdots \times \mathbb{C}}^{k \text { раз }}
$$

представляется в виде произведения $(n-k)$ копий единственной кривой $\mathcal{C}$ и $k$ копий $\mathbb{C}$.

Мы выделили эти два класса интегрируемых систем потому, что в большинстве современных методов построения и изучения вполне интегрируемых систем интегралы движения определяются как модули одной единственной плоской кривой $\mathcal{C}$. При этом данная кривая может быть либо спектральной кривой матришы Лакса [3], либо функцией Казимира для пуассонова пучка, зависяшего от параметра [4].

Перейдем теперь к обычному координатному описанию метода разделения переменных. Пусть на многообразии $\mathcal{M}$ задана система канонических координат $\left\{p_{i}, q_{i}\right\}_{i=1}^{n}$. Интегралы движения $I_{j}(p, q)$ являются функциями этих исходных физических переменных. Предположим, что существует каноническое преобразование $(p, q) \mapsto(P, Q)$ фазового пространства $\mathcal{M}$ такое, что пары канонических переменных разделения $\left(P_{j}, Q_{j}\right)$ являются точками плоских кривых $\mathcal{C}_{j}(1.2)$, т.е.

$$
\Phi_{j}\left(P_{j}, Q_{j}, \alpha_{1}, \ldots, \alpha_{n}\right)=0, \quad j=1, \ldots, n .
$$

Модули кривых отождествляются со значениями интегралов движения $\alpha_{j}=I_{j}$. Подставляя в уравнения плоских кривых вместо значений интегралов движения сами функции, получим уравнения

$$
\Phi_{j}\left(P_{j}, Q_{j}, I_{1}(P, Q), \ldots, I_{n}(P, Q)\right)=0, \quad j=1, \ldots, n,
$$

которые называются разделенными уравнениями.

Переменные разделения могут быть введены явно, например как функции от исходных физических переменных

$$
Q_{i}=f_{i}(p, q), \quad P_{i}=g_{i}(p, q), \quad i=1, \ldots, n,
$$


или с помошью производящей функции соответствующего канонического преобразования. С другой стороны, переменные разделения могут быть заданы неявно, например как полюсы функции Бейкера-Ахиезера [5] или собственные значения тензора Нийенхейса [6].

Подчеркнем, что величины $\mu$ и $\lambda$ являются комплексными переменными, связанными соотношением (1.2), в которое входят числа $\alpha_{j}$. Переменные разделения $P_{i}$ и $Q_{i}$ в уравнении (1.3) являются каноническими переменными в пространстве $\mathcal{M}$. Необходимо помнить, что при переходе от уравнений плоских кривых (1.2) к разделенным уравнениям (1.4) координаты $\lambda=\psi\left(P_{i}, Q_{i}\right)$ и $\mu=\phi\left(P_{i}, Q_{i}\right)$ должны быть функциями от $P_{i}, Q_{i}$. Всюду далее мы не будем выделять это в обозначениях.

Таким образом, в методе разделения переменных сушествуют две взаимосвязанные проблемы. Во-первых, необходимо представить лагранжево многообразие $\mathcal{C}^{(n)}$ в виде произведения (1.1) плоских кривых. Во-вторых, необходимо ввести канонические переменные разделения, удовлетворяющие разделенным уравнениям (1.4).

В данной работе предложен метод явного построения переменных разделения (1.5) для тех интегрируемых систем, для которых известны уравнения плоских кривых (1.2). Эти уравнения можно получить, например, используя сингулярный анализ уравнений движения [7], [8], бигамильтонову структуру интегрируемых систем [8], [9], представление уравнений движения в форме Лакса [5] и некоторые другие методы [10].

\section{2. ПОСТРОЕНИЕ ПЕРЕМЕННЫХ РАЗДЕЛЕНИЯ}

Начнем с простого примера. Для задачи Кеплера лагранжево многообразие $\mathcal{C}^{(2)}=$ $\mathcal{C}_{1} \times \mathcal{C}_{2}$ зависит от значений интегралов движения

$$
I_{1}=p_{x}^{2}+p_{y}^{2}+\frac{Z}{\sqrt{x^{2}+y^{2}}}, \quad I_{2}=2 p_{x}\left(p_{x} y-p_{y} x\right)+\frac{Z y}{\sqrt{x^{2}+y^{2}}}
$$

и величины заряда $Z$. Подставим в уравнения кривых

$$
\mathcal{C}_{1,2}: \quad \Phi_{1,2}(\mu, \lambda)=\left(\mu^{2}+\alpha_{1} \lambda+Z \pm \alpha_{2}\right)=0, \quad I_{1}=\alpha_{1}, \quad I_{2}=\alpha_{2}
$$

функции $I_{k}\left(p_{x}, x, p_{y}, y\right)$ вместо их значений $\alpha_{k}$ и продифференцируем эти уравнения по $Z$. Решения полученных уравнений являются искомыми переменными разделения,

$$
\frac{\partial}{\partial Z} \Phi_{1,2}(\mu, \lambda)=0 \quad \Leftrightarrow \quad \lambda_{1,2}=y \pm \sqrt{x^{2}+y^{2}} .
$$

Таким образом, в данном примере переменные разделения не зависят от заряда $Z$ и их можно построить, используя этот параметр.

Предположим сначала, что у нас есть некоторое семейство лагранжевых многообразий, зависяшее от $(n+m)$ параметров. Произвольные $n$ параметров $\alpha_{1}, \ldots, \alpha_{n}$ отождествляются со значениями интегралов движения $I_{j}=\alpha_{j}$ [1]. Остаюшиеся константы 
$a_{1}, \ldots, a_{m}$ входят в определение интегралов движения $I_{j}$ как произвольные параметры (заряды):

$$
I_{j}(p, q, a)=I_{j}\left(p_{1}, \ldots, p_{n}, q_{1}, \ldots, q_{n}, a_{1}, \ldots, a_{m}\right), \quad a_{k} \in \mathbb{R} .
$$

Основная идея предлагаемого метода состоит в использовании остающихся произвольных параметров $a_{1}, \ldots, a_{m}$ при построении переменных разделения для выбранного нами семейства интегралов движения $I_{j}=\alpha_{j}$.

Предположим теперь, что нам известно представление многообразия $\mathcal{C}^{(n)}$ в виде произведения (1.1) плоских кривых. Подставим функции $I_{j}(p, q)$ вместо их значений $\alpha_{j}$ в уравнения кривых (1.2),

$$
\Phi_{j}\left(\mu, \lambda, I_{1}(p, q, a), \ldots, I_{n}(p, q, a), a_{1}, \ldots, a_{m}\right)=0, \quad j=1, \ldots, n .
$$

Получающиеся уравнения можно дополнить, дифференцируя данные уравнения по параметрам $a_{k}$,

$$
\frac{\partial}{\partial a_{k}} \Phi_{j}\left(\mu, \lambda, I_{1}(p, q, a), \ldots, I_{n}(p, q, a), a_{1}, \ldots, a_{m}\right)=0, \quad k=1, \ldots, m,
$$

или подставляя в них другие значения параметров, например $a_{k}=0$,

$$
\left.\Phi_{j}\left(\mu, \lambda, I_{1}(p, q, a), \ldots, I_{n}(p, q, a), a_{1}, \ldots, a_{m}\right)\right|_{a=0}=0, \quad j=1, \ldots, n
$$

ПРЕДЛОЖЕНИЕ 1. Если каноническое преобразование $(p, q) \mapsto(P, Q)$ не зависит от произвольных параметров $a_{k}$, то решения систем уравнений (2.2), (2.3) или (2.2)-(2.4) являются координатами разделения $\left\{P_{j}, Q_{j}\right\}$, выраженными в виде функций (1.5) от исходных физических переменных $\left\{p_{j}, q_{j}\right\}$.

Обсудим возможность использования данного свойства переменных разделения для явного построения этих переменных.

Пусть рассматриваемая интегрируемая система не зависит от дополнительных параметров, но нам известны и уравнения плоских кривых (1.2), и каноническое преобразование $(p, q) \rightarrow(P, Q)$. Введем новые плоские кривые вида

$$
\mathcal{C}_{j}^{a}: \Phi_{j}^{a}\left(\lambda, \mu, \alpha_{1}, \ldots, \alpha_{n}, a_{1}, \ldots, a_{n}\right)=\Phi_{j}\left(\lambda, \mu, \alpha_{1}, \ldots, \alpha_{n}\right)+a_{j} \lambda=0,
$$

где $\Phi\left(\lambda, \mu, \alpha_{1}, \ldots, \alpha_{n}\right)$ - функции, определяющие исходные кривые (1.2). Произведение данных кривых $\mathcal{C}_{1}^{a} \times \cdots \times \mathcal{C}_{n}^{a}$ является $2 n$-параметрическим лагранжевым многообразием. Применяя обратное каноническое преобразование $(P, Q) \rightarrow(p, q)$, которое не зависит от параметров $a_{j}$, можно отождествить параметры $\alpha_{k}$ с новыми функциями $I_{k}^{a}$ на пространстве $\mathcal{M}$.

Таким образом, для любой интегрируемой системы с интегралами $I_{j}$, допускающей разделение переменных, существует семейство интегрируемых обобщений такое, что $\lim _{a \rightarrow 0} I_{j}^{a}=I_{j}$. Это семейство можно использовать для нахождения переменных разделения с помощью уравнений (2.2), (2.3). 
Итак, мы доказали существование интегрируемых обобщений исходной системы, позволяющих находить переменные разделения. Основной вопрос состоит в том, как построить эти обобщения в исходных физических переменных. Ответа на этот вопрос мы не знаем. Однако можно добавлять параметры, используя канонические преобразования исходных переменных. Можно также рассматривать построенные другими методами обобшения данной системы, которые зависят от параметров и не сильно изменяют уравнения плоских кривых.

Пусть нам известно некоторое обобщение исходной интегрируемой системы, в которое входят параметры $a_{k}$. Необходимо проверить, что искомое каноническое преобразование не зависит от этих параметров. Для этого достаточно решить уравнения $(2.2),(2.3)$ и проверить независимость решений от параметров $a_{k}$.

В некоторых частных случаях ситуация упрошается. Например, если уравнения кривых имеют вид

$$
\Phi_{j}(\mu, \lambda, \alpha, a)=G_{j}(\mu, \lambda)+F_{j}(\lambda, \alpha, a),
$$

то для того чтобы искомое каноническое преобразование не зависело от параметра $a_{k}$, необходимо выполнение дополнительного условия

$$
\frac{\partial^{2} \Phi_{j}(\mu, \lambda)}{\partial a_{k}^{2}}=\frac{\partial^{2} F_{j}(\lambda, I, a)}{\partial a_{k}^{2}}=0 .
$$

С другой стороны, как мы покажем далее, для квазиоднородных интегрируемых систем на орбитах коприсоединенного представления групп Ли ситуация также упрощается. В этом случае операторы Казимира не только порождают семейство интегралов в инволюции, но и позволяют построить переменные разделения для данного семейства интегралов.

\section{3. ОДНОРОДНЫЕ СИСТЕМЫ}

3.1. Система Энона-Эйлеса. Начнем с рассмотрения интегрируемой системы Энона-Эйлеса, которая неоднократно использовалась как модельный пример в рамках различных подходов к построению решений уравнения Гамильтона-Якоби [8], [9], [11].

Рассмотрим однородную интегрируемую систему с функцией Гамильтона

$$
H=T+V=\frac{p_{x}^{2}}{2}+\frac{p_{y}^{2}}{2}-x\left(y^{2}+\frac{1}{3} x^{2}\right) .
$$

Соответствуюшая плоская кривая $\mathcal{C}_{1,2}(1.2)$ в разложении двумерного лагранжева многообразия $\mathcal{C}^{(2)}=\mathcal{C} \times \mathcal{C}$ определяется уравнением

$$
\mathcal{C}: \quad \Phi(\lambda, \mu)=\mu^{2}-\lambda^{3}-\frac{\alpha_{1}}{2}-\frac{\alpha_{2}}{4 \lambda}=0 .
$$

Эта гиперэллиптическая кривая совпадает со спектральной кривой $(2 \times 2)$-матрищы Лакса [11] и может быть получена либо методами сингулярного анализа [8], либо при изучении бигамильтоновой структуры данной системы [9].

3 Теоретическая и математическая физика, т. 128, № 2, 2001 г. 
Соответствующее двумерное лагранжево многообразие $\mathcal{C}^{(2)}$ зависит только от значений двух интегралов движения. Используя масштабное преобразование переменных $(x, y) \mapsto(a x, a y)$, можно построить функцию Гамильтона и второй интеграл движения

$$
H=\frac{p_{x}^{2}}{2}+\frac{p_{y}^{2}}{2}-a x\left(y^{2}+2 x^{2}\right), \quad I=\left(x p_{y}-y p_{x}\right) p_{y}+a \frac{y^{2}}{4}\left(4 x^{2}+y^{2}\right),
$$

которые зависят от одного параметра $а$. Данное преобразование не изменяет рода и положения особых точек исходной гиперэллиптической кривой первого рода

$$
\mathcal{C}^{a}: \Phi(\lambda, \mu)=\mu^{2}-a \lambda^{3}-\frac{\alpha_{1}}{2}+\frac{\alpha_{2}}{4 \lambda}=0 .
$$

Подставляя функцию Гамильтона и второй интеграл движения вместо модулей $\alpha_{1}=H$ и $\alpha_{2}=I$ гиперэллиптической кривой (3.4) и дифференцируя полученное уравнение (2.2) по параметру $а$, построим уравнение вида (2.3),

$$
\frac{\partial \Phi(\lambda, \mu)}{\partial a}=0 \Leftrightarrow\left(4 x^{2}+y^{2}+4 x \lambda+4 \lambda^{2}\right)\left(4 \lambda^{2}-4 x \lambda+y^{2}\right)=0
$$

которое не зависит от параметра $a$ и поэтому определяет координаты разделения. Однако уравнение (3.5) имеет четыре различных решения вместо необходимых двух. Подставив данные решения в уравнение кривой и вычислив соответствующие импульсы $\mu_{j}$, можно выделить две искомые координаты $\lambda_{j}=Q_{j}$, используя условие независимости получаемых импульсов от параметра $a$.

Можно рассмотреть и другое интегрируемое обобшение исходной системы

$$
H_{\mathrm{g}}=H-b\left(2 x^{2}-\frac{y^{2}}{2}\right)-2 c x, \quad I_{\mathrm{g}}=I+b x y^{2}+c y^{2},
$$

которое связано с другой реализацией исходной гиперэллиптической кривой $\mathcal{C}$,

$$
\mathcal{C}^{a b c}: \quad \mu^{2}-a \lambda^{3}-b \lambda^{2}-c \lambda-\frac{\alpha_{1}}{2}-\frac{\alpha_{2}}{4 \lambda}=0 .
$$

Подставляя в уравнение (3.7) интегралы движения и дифференцируя по параметрам $b$ и $c$, дополним единственное уравнение (3.5) до системы уравнений (2.3),

$$
\begin{aligned}
& \frac{\partial \Phi(\lambda, \mu)}{\partial b}=0 \quad \Leftrightarrow \quad(\lambda+x)\left(4 \lambda^{2}-4 x \lambda-y^{2}\right)=0, \\
& \frac{\partial \Phi(\lambda, \mu)}{\partial c}=0 \quad \Leftrightarrow \quad 4 \lambda^{2}-4 x \lambda-y^{2}=0 .
\end{aligned}
$$

Нули наибольшего обшего делителя полученных уравнений

$$
e(\lambda)=\lambda-x-\frac{y^{2}}{4 \lambda}=0
$$

не зависят от параметров и являются переменными разделения $Q_{1,2}$. Таким образом, для определения переменных разделения необходимо использовать как минимум два дополнительных параметра. 
В функцию Гамильтона $H_{\mathrm{g}}(3.6)$ можно добавить еще одно слагаемое,

$$
H_{\mathrm{g}} \mapsto H_{\mathrm{g}}-\frac{a d}{2} y^{2},
$$

которое приводит к изменению соответствующей гиперэллиптической кривой

$$
\mathcal{C}^{d}: \quad \mu^{2}=a \lambda^{3}+b \lambda^{2}+c \lambda+\frac{2 \alpha_{1} \lambda-\alpha_{2}}{4(\lambda-d)} .
$$

При этом уравнения вида (2.3) явно зависят от параметра $d$, т.е. нарушается условие (2.6). Таким образом, надо исключить соответствующее уравнение из системы (2.3). Нули наибольшего обшего делителя оставшихся уравнений типа (2.3) определяются уравнением

$$
e(\lambda)=\lambda-x-\frac{y^{2}}{\lambda-d}=0,
$$

и точечное преобразование $(x, y) \mapsto\left(Q_{1}, Q_{2}\right)$ явно зависит от параметра $d$.

3.2. Штеккелевские системы. Напомним, что для всех штеккелевских систем уравнения кривых имеют вид (2.5) [10], и все интегрируемые системы на римановых пространствах постоянной кривизны, допускающие разделение переменных в криволинейных ортогональных системах координат, принадлежат штеккелевскому семейству интегрируемых систем [12].

Вполне интегрируемые штеккелевские системы [10] в пространстве $\mathbb{R}^{2 n}$ однозначно определяются невырожденной $(n \times n)$-матрицей Штеккеля $\mathbf{S}$, элементы $j$-го столбца которой $s_{k j}$ зависят лишь от координаты $q_{j}$,

$$
\operatorname{det} \mathbf{S} \neq 0, \quad \frac{\partial s_{k j}}{\partial q_{m}}=0, \quad j \neq m .
$$

Данной матрице $\mathbf{S}$ сопоставим $n$ квадратичных по импульсам функционально независимых интегралов движения $I_{k}, k=1, \ldots, n$, находяшихся в инволюции $\left\{I_{k}, I_{j}\right\}=0$, по правилу

$$
I_{k}=\sum_{j=1}^{n} c_{j k}\left(p_{j}^{2}+U_{j}\left(q_{j}\right)\right), \quad c_{j k}=\frac{\mathbf{S}^{k j}}{\operatorname{det} \mathbf{S}} .
$$

Здесь $\mathbf{C}=\left[c_{j k}\right]$ - матрица, обратная к матрице Штеккеля $\mathbf{S}$, a $\mathbf{S}^{k j}$ - алгебраическое дополнение элемента $s_{k j}$ в определителе $\operatorname{det} \mathbf{S}$.

Совместная поверхность уровня интегралов $I_{k}(3.10)$

$$
M_{\alpha}=\left\{z \in \mathbb{R}^{2 n}: I_{k}(z)=\alpha_{k}, k=1, \ldots, n\right\}
$$

диффеоморфна $n$-мерному тору [2], откуда сразу же следует, что

$$
p_{j}^{2}=\left(\frac{\partial \mathcal{S}_{0}}{\partial q_{j}}\right)^{2}=\sum_{k=1}^{n} \alpha_{k} s_{k j}\left(q_{j}\right)-U_{j}\left(q_{j}\right),
$$


где $\mathcal{S}_{0}\left(q_{1}, \ldots, q_{n}\right)$ - функция укороченного действия [2]. Римановы поверхности (3.12) изоморфны каноническим гиперэллиптическим кривым

$$
\mathcal{C}_{j}: \quad \mu^{2}-F_{j}(\lambda)=\mu^{2}-\prod_{i=1}^{k}\left(\lambda-e_{i}\right)=0,
$$

где модули кривой $e_{i}$ являются функциями от значений интегралов движения $\alpha_{i}$. Все вместе алгебраические кривые $\mathcal{C}_{j}$ определяют $n$-мерное лагранжево подмногообразие полного симплектического многообразия

$$
\mathcal{C}^{(n)}: \quad \mathcal{C}_{1}\left(p_{1}, q_{1}\right) \times \mathcal{C}_{2}\left(p_{2}, q_{2}\right) \times \cdots \times \mathcal{C}_{n}\left(p_{n}, q_{n}\right),
$$

где импульсы находятся из уравнения (3.12).

ПРедЛожениЕ 2. Разложим штеккелевские потенциаль $U_{j}\left(q_{j}\right)$ в ряд Лорана:

$$
U_{j}\left(q_{j}\right)=\sum a_{k j} q_{j}^{k}, \quad a_{k j} \in \mathbb{R} .
$$

Так как интеграль движения $I_{i}(3.10)$ зависят от коэффициентов ряда $a_{k j}$, то переменные разделения $q_{i}$ являются решениями системы уравнений (2.3), которая в данном случае имеет вид

$$
\frac{\partial F_{j}}{\partial a_{k j}}=0 \Rightarrow \lambda^{k}-q_{j}^{k}=0, \quad j=1, \ldots, n, \quad k=1,2, \ldots .
$$

Сопряженные им импульсы находятся из уравнений (2.2), которые в данном случае имеют вид (3.12).

Элементы матрицы Штеккеля также могут зависеть от произвольных параметров $d_{1}, \ldots, d_{m}$. Однако при этом не вьполняется необходимое для штеккелевских кривых дополнительное условие (2.6), и тем самым искомое каноническое преобразование явно зависит от параметров $d$.

3.3. Периодическая $D_{n}$-цепочка Тоды. Рассмотрим обобщенную цепочку Тоды, связанную с системой корней $D_{n}$. Соответствуюшая функция Гамильтона имеет вид

$$
H=\sum_{j=1}^{n}\left(\frac{1}{2} p_{j}^{2}+e^{q_{j+1}-q_{j}}\right)+e^{-q_{n-1}-q_{n}}+e^{q_{1}+q_{2}} .
$$

Уравнения движения обладают $n$ интегралами движения, которые были получены как спектральные инварианты матрицы Лакса размерности $(2 n \times 2 n)$ [13], [14]. Интегралы движения и спектральная кривая матрицы Лакса зависят только от $n$ значений интегралов движения,

$$
\mathcal{C}: \quad \mu+\frac{\lambda^{4}}{\mu}+\lambda^{2 n}+2 \alpha_{1} \lambda^{2 n-2}+\alpha_{2} \lambda^{2 n-4}+\cdots=0, \quad \alpha_{1}=H .
$$


Перейдем к рассмотрению обобщенной цепочки Тоды типа $D_{n}$, предложенной Иноземцевым [14],

$$
H_{\mathrm{g}}=H+\frac{a}{\operatorname{sh}^{2} q_{n}}+\frac{b}{\operatorname{sh}^{2} \frac{q_{n}}{2}}+\frac{c}{\operatorname{sh}^{2} q_{1}}+\frac{d}{\operatorname{sh}^{2} \frac{q_{1}}{2}}, \quad a, b, c, d \in \mathbb{R} .
$$

Новое уравнение плоской кривой имеет вид

$$
\begin{aligned}
\Phi(\mu, \lambda)= & \mu+\frac{\lambda^{2}\left(\lambda^{4}+(2 b-a) \lambda^{2}+b^{2}\right)\left(\lambda^{4}+(2 c-d) \lambda^{2}+d^{2}\right)}{\mu}+ \\
& +\lambda^{2 n+2}+2 \alpha_{1} \lambda^{2 n}+\alpha_{2} \lambda^{2 n-2}+\cdots=0, \quad \alpha_{1}=H_{\mathrm{g}} .
\end{aligned}
$$

Дифференцируя данное уравнение по $a$ и $b$ или по $c$ и $d$, получим две системы уравнений

$$
\Phi(\mu, \lambda)=0, \quad \frac{\partial \Phi(\mu, \lambda)}{\partial a}=0, \quad \frac{\partial \Phi(\mu, \lambda)}{\partial b}=0
$$

или

$$
\Phi(\mu, \lambda)=0, \quad \frac{\partial \Phi(\mu, \lambda)}{\partial c}=0, \quad \frac{\partial \Phi(\mu, \lambda)}{\partial d}=0 .
$$

Решения первой системы зависят явно от параметров $c$ и $d$, а решения второй системы зависят, в свою очередь, от параметров $a$ и $b$.

ПРЕДЛОЖЕНИЕ 3. Решения систем уравнений (3.19), (3.20) определяют два семейства переменных разделения $\lambda=Q_{j}$ и $\mu=\phi\left(P_{j}\right)$ для иепочки Тоды. Эти семейства связаны каноническим преобразованием, индуцированным автоморфизмом второго порядка пополненного графа Дьнкина $D_{n}-$ типа.

ДоКАЗАТЕЛЬСТво этого утверждения состоит в проверке совпадения решений данных уравнений с полюсами функции Бейкера-Ахиезера с нестандартной динамической нормировкой, которые использовались в работе [15] для построения переменных разделения.

\section{4. КВАЗИОДНОРОДНЫЕ ИНТЕГРИРУЕМЫЕ СИСТЕМЫ}

4.1. Цепочка Тоды. Обычная периодическая цепочка Тоды - это система $n$ взаимодействующих частиц с гамильтонианом

$$
H(p, q)=\frac{1}{2} \sum_{i=1}^{n}\left(p_{i}^{2}+e^{q_{i}-q_{i+1}}\right) .
$$

Здесь $p_{i}, q_{i}-$ координата и импульс $i$-й частицы, т.е. канонические координаты фазового пространства $\mathcal{M}=\mathbb{R}^{2 n}$. Условия периодичности имеют вид $q_{i+n}=q_{i}$ и $p_{i+n}=p_{i}$ для любого индекса $i$.

Соответствующие уравнения движения обладают $n$ интегралами движения, которые были получены впервые из матриц Лакса размерности $(n \times n)$ [13]. В данной работе 
мы будем использовать эквивалентное представление Лакса в виде матриц размерности $(2 \times 2)$

$$
T(\lambda)=L_{n}(\lambda) \ldots L_{2}(\lambda) L_{1}(\lambda), \quad L_{i}=\left(\begin{array}{cc}
\lambda+p_{i} & -e^{q_{i}} \\
e^{-q_{i}} & 0
\end{array}\right) .
$$

Уравнение спектральной кривой $\mathcal{C}: \operatorname{det}(T(\lambda)+\mu)=0$ матришы Лакса (4.2) зависит только от $n$ интегралов движения и не зависит от дополнительных параметров,

$$
\Phi(\mu, \lambda)=\left(\mu+\frac{1}{\mu}\right)+\lambda^{n}+\lambda^{n-1} I_{1}+\lambda^{n-2} I_{2}+\cdots=0 .
$$

Здесь $I_{1}=\sum p_{i}-$ полньй импульс, описываюший движение центра масс, а второй интеграл $I_{2}=I_{1}^{2} / 2-H$ определяет функцию Гамильтона. Эта же гиперэллиптическая кривая может быть получена методами сингулярного анализа [7], [8], а также при исследовании бигамильтоновой геометрии цепочки Тоды [6].

Так как полный импульс системы $I_{1}=\sum p_{i}$ является интегралом движения, то данная система принадлежит к классу квазиоднородных интегрируемых систем

$$
\mathcal{C}^{(n)}: \overbrace{\mathcal{C} \times \cdots \times \mathcal{C}}^{(n-1) \text { раз }} \times \mathbb{R}^{2} .
$$

Для данной модели, используя полюсы функции Бейкера-Ахиезера [5], преобразование Беклунда [16] или собственные значения тензора Нийенхейса [6], можно построить только $2(n-1)$ переменных разделения. Оставшиеся две переменныенам приходится вводить исходя из некоторых дополнительных предположений.

Рассмотрим поверхность уровня $S_{a}$ интеграла $I_{1}=a$, т.е. цепочку Тоды в системе центра масс, которая принадлежит к классу однородных интегрируемых систем. Соответствуюшее $(n-1)$-мерное лагранжево многообразие зависит от $(n-1)$ значений интегралов движения и от одного дополнительного параметра $a$.

ПредЛоЖениЕ 4. Переменные разделения для чепочки Тоды в системе центра масс являются решениями системь уравнений (2.2), (2.3),

$$
\Phi\left(\mu, \lambda, a, \hat{I}_{2}, \ldots, \hat{I}_{n}\right)=0, \quad \frac{\partial}{\partial a} \Phi\left(\mu, \lambda, a, \hat{I}_{2}, \ldots, \hat{I}_{n}\right)=0,
$$

где функичя $\Phi$ определена в (4.3), а $\hat{I}_{j}$ - ограничения интегралов $I_{j}$ на поверхность уровня $S_{a}$ общего положсения, т.е. при произвольных значениях параметра а.

ДокАЗАТЕЛЬСтво. Следуя работе [17], перепишем матришу Лакса в виде

$$
T(\lambda)=\left(\begin{array}{cc}
e^{-\frac{Q}{2}} & 0 \\
0 & e^{\frac{Q}{2}}
\end{array}\right)\left(\begin{array}{cc}
\lambda-P+\sum_{i=1}^{n-1} \widehat{p}_{i} & -1 \\
1 & 0
\end{array}\right) \prod_{i=1}^{n-1}\left(\begin{array}{cc}
\lambda+\hat{p}_{i} & -e^{\hat{q}_{i}} \\
e^{-\hat{q}_{i}} & 0
\end{array}\right)\left(\begin{array}{cc}
e^{\frac{Q}{2}} & 0 \\
0 & e^{-\frac{Q}{2}}
\end{array}\right) .
$$

Здесь $Q$ и $P$ - координата и импульс центра масс, а $\hat{q}_{i}$ и $\hat{p}_{i}$ - соответствующие переменные Якоби. Ограничение данной матрицы и интегралов движения на $S_{a}$ достаточно 
очевидно, и второе из уравнений (4.5) приводится к виду

$$
T_{1,2}(\lambda)=0,
$$

где $T_{1,2}$ - внедиагональный элемент матрицы Лакса $T(\lambda)$. Решения этого уравнения совпадают с полюсами функции Бейкера-Ахиезера, нормированной на вектор $\vec{\alpha}=(1,0)$ [5], [18].

Теперь мы можем вернуться к исходному симплектическому многообразию. Так как по определению интегралы движения находятся в инволюции $\left\{I_{j}, I_{k}\right\}=0$, то скобки Пуассона между полным импульсом $I_{1}=\sum p_{i}$ и решениями системы уравнений (4.5) равны нулю. Добавляя координату $Q$ и импульс $P$ центра масс к решениям системы уравнений (4.5), получаем $2 n$ переменных разделения. При этом дополнительное $n$-е разделенное уравнение имеет вид $P=I_{1}$.

4.2. Старшие стационарные потоки уравнения КдФ. Следуя работе [19], рассмотрим старшие потоки эволюционных уравнений. Хотя все результаты будут справедливы для всех рассмотренных в [19] иерархий эволюционных уравнений, мы ограничимся изучением стационарных потоков уравнения $\mathrm{K} \Phi \Phi$, которые связаны с однородными системами типа Штеккеля [20], [21]

Пусть $\{\mathbf{e}, \mathbf{f}, \mathbf{h}\}$ - базис в алгебре Ли $s l(2)$,

$$
[\mathbf{h}, \mathbf{e}]=\mathbf{e}, \quad[\mathbf{h}, \mathbf{f}]=-\mathbf{f}, \quad[\mathbf{e}, \mathbf{f}]=2 \mathbf{h},
$$

а элемент

$$
\Delta=\mathbf{h}^{2}+\frac{1}{2}(\mathbf{e f}+\mathbf{f e})
$$

обертываюшей алгебры является оператором Лапласа на группе $S L(2)$. Определим представление $\mathcal{W}$ алгебры $s l(2)$ в линейном пространстве $V$ так, что

$$
\mathcal{W}:\{\mathbf{e}, \mathbf{f}, \mathbf{h}\} \rightarrow\{e, f, h\} \in \operatorname{End}(V) .
$$

Используя прямую сумму $(n+1)$ экземпляра алгебры петель $\widetilde{s l(2)}$, мы можем построить многополюсную матрицу Лакса

$$
L_{0}=\left(\begin{array}{ll}
0 & 1 \\
0 & 0
\end{array}\right)+\sum_{j=1}^{n} \frac{1}{2\left(\lambda-d_{j}\right)}\left(\begin{array}{cc}
h_{j} & e_{j} \\
f_{j} & -h_{j}
\end{array}\right)
$$

в рамках метода классической $r$-матрицы [3]. Здесь $\left\{e_{j}, f_{j}, h_{j}\right\}$ - элементы представления в некотором пространстве $V_{j}$. Например, следующая реализация бесконечномерного представления алгебры $\operatorname{sl}(2)$ вида

$$
h_{j}=x_{j} p_{j}, \quad e_{j}=x_{j}^{2}, \quad f_{j}=-p_{j}^{2}+\frac{a_{j}}{x_{j}^{2}}, \quad a_{j} \in \mathbb{R},
$$

связана с обычными стационарными потоками и движением по геодезическим на римановых пространствах со штеккелевской метрикой [20], [21]. 
Чтобы ввести старшие потоки, достаточно использовать так называемую $m$-бозонную реализацию бесконечномерного представления алгебры $s l(2)$ вместо однобозонной реализации (4.9). Например, двухбозонная реализация имеет вид

$$
h_{j}=\frac{1}{2}\left(x_{j} p_{x, j}-y_{j} p_{y, j}\right), \quad e_{j}=x_{j} p_{y, j}, \quad f_{j}=y_{j} p_{x, j}
$$

и связана с так называемыми бинарными стационарными потоками [19].

Одновременно с движением по геодезическим можно рассмотреть и потенциальное движение. Соответствуюшая матрица Лакса записывается в виде

$$
L(\lambda)=\left(\begin{array}{cc}
h & e \\
f & -h
\end{array}\right)(\lambda)=L_{0}(\lambda)+\left(\begin{array}{cc}
0 & 0 \\
{\left[\phi(\lambda) e^{-1}(\lambda)\right]_{M N}} & 0
\end{array}\right) .
$$

Здесь параметрическая функция

$$
\phi(\lambda)=\sum_{-\infty}^{\infty} \phi_{k} \lambda^{k}, \quad \phi_{k} \in \mathbb{R}
$$

определяет потенциал $U(x)$, а скобки $[z]_{M N}$ обозначают линейную комбинацию лорановских проекций [21],

$$
[z]_{M N}=\left[\sum_{k=-\infty}^{+\infty} z_{k} \lambda^{k}\right]_{M N} \equiv \sum_{k=-M}^{N} z_{k} \lambda^{k}
$$

Для коэффициентов параметрической функции $\phi_{k}$ система уравнений $(2.3)$ имеет вид

$$
\frac{\partial}{\partial \phi_{k}} \Phi(\mu, \lambda, \alpha, \phi)=0 \Leftrightarrow \frac{\partial f(\lambda)}{\partial \phi_{k}} e(\lambda)=0 .
$$

Нули наибольшего обшего делителя $e(\lambda)$ совпадают с полюсами функции БейкераАхиезера, нормированной на вектор $\vec{\alpha}=(1,0)$, и определяют $2 n$ переменных разделения. Эта конструкция матриц Лакса и переменных разделения не зависит от конкретной реализации представлений алгебры $s l(2)$.

Тот же самый результат можно получить, используя элементы Казимира $\Delta_{j}(4.7)$. Для первой реализации (4.9) операторы $\Delta_{j}$ равны произвольным параметрам $a_{j}$. Соответствуюшая система уравнений (2.3) принимает вид

$$
\frac{\partial}{\partial a_{k}} \Phi(\mu, \lambda, \alpha, a)=0 \Leftrightarrow \frac{\partial f(\lambda)}{\partial a_{k}} e(\lambda)=0 .
$$

Подставляя вторую реализацию (4.10) в определения матриц Лакса (4.11), мы определим интегрируемые системы на $4 n$-мерном симплектическом многообразии $\mathcal{M}$ с каноническими координатами $\left\{x_{j}, p_{x, j}, y_{j}, p_{y, j}\right\}_{j=1}^{n}$. Первые $n$ штеккелевских интегралов $I_{1}, \ldots, I_{n}$ квадратичны по импульсам (3.10), а оставшиеся $n$ интегралов

$$
I_{n+j}=\frac{\left(x_{j} p_{x, j}+y_{j} p_{y, j}\right)^{2}}{4}, \quad j=1, \ldots, n
$$


совпадают со значениями операторов Казимира $\Delta_{j}(4.7)$. Тем не менее на пространстве $\mathcal{M}$ величины $I_{n+j}$ являются интегралами движения, описываюшими нетривиальную динамику. Так как мы рассматриваем бесконечномерные представления, для которых спектр оператора Казимира непрерывен, то соответствующее $2 n$-мерное лагранжево многообразие имеет вид

$$
\mathcal{C}^{(2 n)}: \overbrace{\mathcal{C} \times \cdots \times \mathcal{C}}^{n \text { раз }} \times \overbrace{\mathbb{R} \times \cdots \times \mathbb{R}}^{n \text { раз }} .
$$

Здесь $\mathcal{C}$ - спектральная кривая матришы Лакса вида

$$
\mathcal{C}: \quad \mu^{2}+P\left(\lambda, I_{1}, \ldots, I_{n}, \phi_{1}, \ldots, \phi_{m}\right)+\sum_{j=1}^{n} \frac{I_{n+j}}{\left(\lambda-d_{k}\right)^{2}}=0
$$

где функция $P$ и интегралы $I_{1}, \ldots, I_{n}$ определяются параметрической функцией $\phi(\lambda)$. Явный вид некоторых кривых можно найти в работе [19].

Соответствуюшая пуассонова структура вырожденна, и функция Бейкера-Ахиезера имеет только $n$ полюсов. Однако на поверхности уровня $S_{b}$ функций $I_{n+j}=b_{j}$ пуассонова структура становится невырожденной, а интегрируемая система - однородной. После редукции $n$-мерное лагранжево многообразие зависит от $n$ значений интегралов $\hat{I}_{j}$, а также от $m$ параметров $\phi_{k}$ и $n$ параметров $b_{j}$.

ПРедЛОЖенИЕ 5. Решения системы уравнений

$$
\Phi\left(\mu, \lambda, \hat{I}_{1}, \ldots, \hat{I}_{n}, \phi, b\right)=0, \quad \frac{\partial}{\partial b_{k}} \Phi\left(\mu, \lambda, \hat{I}_{1}, \ldots, \hat{I}_{n}, \phi, b\right)=0
$$

являются переменными разделения. Здесь $\hat{I}_{j}$ - ограничения интегралов $I_{j}$ на поверхность уровня $S_{b}$ общего положения, т.е. при произвольных $b$.

ДокАЗАТЕльство. Воспользуемся равенствами $\left\{I_{n+j}, e(\lambda)\right\}=\left\{I_{n+j}, h(\lambda)\right\}=0$, установленными в работе [19]. Используя затем редукцию матрицы Лакса на поверхность уровня $S_{b}$, можно доказать, что система уравнений (2.3) имеет вид

$$
\frac{\partial}{\partial b_{k}} \Phi(\mu, \lambda, \alpha, b)=0 \Leftrightarrow \frac{\partial f(\lambda)}{\partial b_{k}} e(\lambda)=0 .
$$

Как и ранее, наибольший обший делитель полученной системы уравнений совпадает с внедиагональным элементом $e(\lambda)$ матрицы Лакса [19].

Так как для построения переменных разделения мы использовали только интегралы движения $(2.2),(2.3)$, то скобки Пуассона между интегралами $I_{n+j}$ и переменными разделения равны нулю. Таким образом, на исходном $4 n$-мерном симплектическом многообразии $2 n$ решений уравнений $(2.2),(2.3)$ и $2 n$ интегралов $I_{n+j}$ вместе с сопряженными величинами образуют полный набор переменных разделения. Для этих дополнительных переменных разделенные уравнения имеют вид $P_{n+j}=I_{n+j}$. 
4.3. Волчок Горячева-Чаплыгина. Отождествим шестимерное пространство $e^{*}(3)$ с пространством $e(3)=s o(3) \oplus \mathbb{R}^{3}$, которое является полупрямой суммой алгебры $s o(3)$ и абелева идеала $\mathbb{R}^{3}$. Введем $J \in s o(3) \simeq \mathbb{R}^{3}$ и $x \in \mathbb{R}^{3}$ - координаты в пространстве $e^{*}(3)$, снабженном естественной скобкой Ли-Пуассона

$$
\left\{J_{i}, J_{j}\right\}=\varepsilon_{i j k} J_{k}, \quad\left\{J_{i}, x_{j}\right\}=\varepsilon_{i j k} x_{k}, \quad\left\{x_{i}, x_{j}\right\}=0,
$$

где $\varepsilon_{i j k}$ - стандартный полностью кососимметричный тензор. Представления алгебры Ли $е(3)$ фиксируются значениями двух операторов Казимира

$$
I_{1}=(x, x)=x_{i} x_{i}, \quad I_{2}=(J, x)=J_{i} x_{i} .
$$

В отличие от двух предыдущих примеров эти операторы описывают тривиальную динамику $\left\{I_{1,2}, x_{k}\right\}=\left\{I_{1,2}, J_{k}\right\}=0$. Напомним, что орбиты $\mathcal{O}\left(I_{1}=c_{1}, I_{2}=c_{2}\right)$ коприсоединенного представления $E(3)$ в $e^{*}(3)$ являются четырехмерными симплектическими многообразиями, инвариантными относительно уравнений Эйлера-Пуассона [2]. Данные многообразия топологически эквивалентны кокасательному расслоению $T^{*} S^{2}$ к двумерной сфиере $S^{2}$.

На исходном шестимерном пространстве $e^{*}(3)$ определим интегралы движения для волчка Горячева-Чаплыгина:

$$
I_{3}=J_{1}^{2}+J_{2}^{2}+4 J_{3}^{2}+x_{2}, \quad I_{4}=2 J_{3}\left(J_{1}^{2}+J_{2}^{2}\right)-x_{3} J_{2} .
$$

Данная система является полностью интегрируемой только на однопараметрическом семействе орбит $\mathcal{O}\left(I_{1}=\right.$ const, $\left.I_{2}=0\right)$.

В исходном шестимерном пространстве соответствуюшее некомпактное лагранжево многообразие имеет вид

$$
\mathcal{C}^{(3)}=\mathcal{C} \times \mathcal{C} \times \mathbb{R} \cup\{0\},
$$

а единственная гиперэллиптическая кривая задается уравнением

$$
\mathcal{C}: \quad \mu \lambda^{2}+\frac{\alpha_{1} \mu+2 x_{3} \alpha_{2}}{\lambda^{2}}+\mu^{3}-2 \alpha_{3} \mu-\alpha_{4}=0, \quad \alpha_{j}=I_{j}, \quad j=1,2,3,4 .
$$

Уравнение плоской кривой (4.16) было получено методами сингулярного анализа [8], [22] и в виде спектральной кривой различных матриц Лакса [23], [24].

Поскольку в данной задаче оператор Казимира $I_{2}$ имеет конкретное значение, а второй оператор $I_{1}$ квадратичен, построить редукцию остающихся интегралов $I_{3}$ и $I_{4}$ на четырехмерные симплектические листы $\mathcal{O}$ достаточно сложно. Поэтому рассмотрим масштабное преобразование $x \rightarrow a x$, которое связывает различные симплектические листы $\mathcal{O}$ так, что $I_{1} \rightarrow a^{2} I_{1}$ и $I_{2} \rightarrow a I_{2}$. Дополнительно будем использовать симметрию данной задачи относительно врашения плоскости, перпендикулярной третьей оси, вида $x_{1,2} \rightarrow x_{1} \sin \phi \pm x_{2} \cos \phi$.

Итак, масштабное преобразование и врашение переводят исходные интегралы (4.15) в интегралы

$$
I_{3}=\frac{1}{2}\left(J_{1}^{2}+J_{2}^{2}+4 J_{3}^{2}\right)+a x_{1}+b x_{2}, \quad I_{4}=2 J_{3}\left(J_{1}^{2}+J_{2}^{2}\right)-\left(a J_{1}+b J_{2}\right) x_{3} .
$$


Соответствующая матрица Лакса

$$
L(\lambda)=\left(\begin{array}{ccc}
-2 J_{3} & -\frac{x_{3}}{\lambda} & -b_{+} \lambda+\frac{x_{+}}{\lambda} \\
\frac{x_{3}}{\lambda} & 0 & J_{+} \\
b_{-} \lambda-\frac{x_{-}}{\lambda} & J_{-} & 2 J_{3}
\end{array}\right),
$$

где

$$
x_{ \pm}=x_{1} \pm i x_{2}, \quad J_{ \pm}=J_{1} \pm i J_{2}, \quad b_{ \pm}=a \pm i b,
$$

является обобщением матрицы Лакса, построенной в работе [24]. Спектральная кривая $\mathcal{C}^{a b}$ данной матрицы имеет вид

$$
\Phi(\mu, \lambda)=\mu \lambda^{2}\left(a^{2}+b^{2}\right)+\frac{I_{1} \mu+2 x_{3} I_{2}}{\lambda^{2}}+\mu^{3}-2 I_{3} \mu-I_{4}=0 .
$$

Таким образом, на каждом симплектическом листе $\mathcal{O}\left(I_{1}=\right.$ const, $\left.I_{2}=0\right)$ двумерное лагранжево многообразие зависит от двух значений интегралов движения $I_{3}, I_{4}$ и двух свободных параметров $b_{+}$и $b_{-}$.

ПРеДЛОЖЕНИЕ 6. Решения системы уравнений

$$
\begin{gathered}
\Phi\left(\mu, \lambda, I_{1}(J, x), \ldots, I_{4}(J, x), b_{+}, b_{-}\right)=0, \\
\frac{\partial \Phi\left(\mu, \lambda, I_{1}(J, x), \ldots, I_{4}(J, x), b_{+}, b_{-}\right)}{\partial b_{+}}=0, \\
\frac{\partial \Phi\left(\mu, \lambda, I_{1}(J, x), \ldots, I_{4}(J, x), b_{+}, b_{-}\right)}{\partial b_{-}}=0
\end{gathered}
$$

являются переменными разделения.

ДокАзАтельство. Решая последние два уравнения относительно переменной $\lambda$ и подставляя полученные решения в первое уравнение, получим два уравнения, в которые входит только переменная $\mu$,

$$
\begin{gathered}
\left(\mu^{2} x_{-}+\mu\left(J_{-} x_{3}-2 x_{-} J_{3}\right)-2 J_{-} J_{3} x-3+b_{-} x_{3}^{2}\right)\left(\mu^{2}-2 J_{3} \mu-J_{1}^{2}-J_{2}^{2}\right)=0 \\
\left(\mu^{2} x_{+}+\mu\left(J_{+} x_{3}-2 x_{+} J_{3}\right)-2 J_{+} J_{3} x-3+b_{+} x_{3}^{2}\right)\left(\mu^{2}-2 J_{3} \mu-J_{1}^{2}-J_{2}^{2}\right)=0 .
\end{gathered}
$$

Нули наибольшего общего делителя полученных уравнений

$$
e(\mu)=\mu^{2}-2 J_{3} \mu-J_{1}^{2}-J_{2}^{2}=0
$$

совпадают с полюсами соответствующей функции Бейкера-Ахиезера, нормированной на вектор $\vec{\alpha}=(1,0,0)$, а система уравнений (4.19) эквивалентна системе уравнений

$$
\operatorname{rank}\left(\begin{array}{c}
\vec{\alpha} \\
L(\mu, \lambda)
\end{array}\right)=2,
$$

определяющей переменные разделения в методе Склянина [5]. Более того, часть уравнений из системы (4.19) совпадает с уравнениями из системы (4.22).

Заметим, что для определения переменных разделения достаточно использовать только одно из двух последних уравнений (4.19), так как в любом из уравнений (4.20) можно явно выделить два решения, которые не зависят от параметров. 
4.4. Система Неймана. Система Неймана описывает движение точки на сфере $\mathcal{S}^{n-1}$ в поле квадратичного потенциала (все необходимые ссылки могут быть найдены в работе [3]). В канонических переменных $x, p$ на $T^{*} \mathcal{S}^{n-1}$ соответствуюшая матрица Лакса имеет вид

$$
L(\lambda)=A+J \lambda+b X \lambda^{2}, \quad J=p \wedge x, \quad X=x \otimes x, \quad b \in \mathbb{R},
$$

где $A$ - произвольная симметрическая числовая матрища, а $b$ - радиус сферы $\mathcal{S}^{n-1}$. Используя врашения сферы, можно привести матрицу $A$ к диагональному виду $A=$ $-\operatorname{diag}\left(a_{1}, \ldots, a_{n}\right)$. При этом функция Гамильтона для системы Неймана имеет вид

$$
H=\frac{1}{2} \sum p_{j}^{2}+b \sum a_{j} x_{j}^{2} .
$$

Ограничимся случаем $n=3$ и отождествим фазовое пространство с объединением однопараметрических орбит $\mathcal{O}\left(I_{1}=\right.$ const, $\left.I_{2}=0\right)$ алгебры $e^{*}(3)$. Как и для волчка Горячева-Чапльгина, вместо редукшии фазового пространства рассмотрим различные симплектические листы общего положения, отвечающие различным радиусам $I_{1}=r^{2}=b$ сферы $\mathcal{S}^{2}$.

ПредЛОЖЕниЕ 7. Решения системы уравнений типа (2.2), (2.3)

$$
\operatorname{det}(L(\lambda)-\mu)=0, \quad \frac{\partial \operatorname{det}(L(\lambda)-\mu)}{\partial b}=0
$$

являются переменными разделения для системь Неймана.

ДОКАЗАТЕЛЬСтво. Вместо последнего уравнения можно использовать уравнение типа (2.4)

$$
\left.\operatorname{det}(L(\lambda)-\mu)\right|_{b=0}=\operatorname{det}(L(\lambda)-\mu)-b \frac{\partial \operatorname{det}(L(\lambda)-\mu)}{\partial b}=0 .
$$

Заметим, что это уравнение определяет спектральную кривую для волчка Эйлера. Исключая переменную $\lambda$, получим известное определение

$$
\prod_{j=1}^{3}\left(\mu-a_{j}\right)\left(\frac{x_{1}^{2}}{\mu-a_{1}}+\frac{x_{2}^{2}}{\mu-a_{2}}+\frac{x_{3}^{2}}{\mu-a_{3}}\right)=0
$$

для эллиптических сфероидальных координат [12], которые являются переменными разделения для системы Неймана [3].

4.5. Стационарные потоки уравнения Буссинеска. Уравнение Буссинеска в рамках общей теории стационарных потоков $n$-иерархий Гельфанда-Дикого изучалось в работе [25]. Следуя этой работе, рассмотрим стационарный поток пятого порядка $t_{5}$ на десятимерном фазовом пространстве $\mathcal{M}$ с координатами $h_{1}, k_{1}, h_{2}, k_{2}, \ldots, h_{5}, k_{5}$, для 

которых тензор Пуассона имеет вид

$$
P_{0}=\left(\begin{array}{cccccccccc}
0 & 0 & 0 & 0 & 0 & 0 & 0 & 0 & -1 & 0 \\
& 0 & 0 & 0 & 0 & 1 & 0 & 0 & 0 & 0 \\
& & 0 & 0 & 0 & 1 & 1 & 0 & 0 & 0 \\
& & & 0 & 1 & 0 & 0 & 0 & 0 & 0 \\
& & & & 0 & 0 & 0 & -h_{1} & -2 h_{1} & -k_{1}-h_{2} \\
& & & & & 0 & -h_{1} & -2 k_{1} & -k_{1}-h_{2} & -2 k_{2} \\
& & & & & & 0 & 0 & -2 h_{2} & -k_{2} \\
& & & & & & & 0 & -k_{2} & 0 \\
& & & & & & & & 0 & 0 \\
& & & & & & & & 0
\end{array}\right) .
$$

Данная пуассонова структура вырожденна, и функции Казимира

$$
I_{1}=3 k_{5}-3 k_{1} k_{2}-3 k_{2} h_{2}, \quad I_{3}=-3 h_{1} k_{2}+3 k_{4}-3 k_{1}^{2}
$$

описывают тривиальную динамику

$$
\left\{I_{1,3}, h_{j}\right\}=\left\{I_{1,3}, k_{j}\right\}=0, \quad j=1,2, \ldots, 5 .
$$

Матрица Лакса [3], [25], вычисленная с использованием представления нулевой кривизны для исходных эволюционных уравнений или построенная в рамках метода классической $r$-матришы, имеет следующий явный вид:

$$
\begin{gathered}
L(\lambda)=\lambda^{2}\left(\begin{array}{ccc}
0 & 0 & 0 \\
1 & 0 & 0 \\
0 & 1 & 0
\end{array}\right)+\lambda\left(\begin{array}{ccc}
0 & 0 & 1 \\
h_{2} & h_{1} & 0 \\
-h_{3}+k_{2} & k_{1}-h_{2} & -h_{1}
\end{array}\right)+ \\
+\left(\begin{array}{ccc}
-k_{3} & -k_{2} & -k_{1} \\
L_{0}^{2,1} & -h_{2} h_{1}+h_{4} & -h_{1}^{2}+h_{3} \\
L_{0}^{3,1} & L_{0}^{3,2} & h 2 h_{1}-h_{4}+k_{3}
\end{array}\right),
\end{gathered}
$$

где

$$
\begin{aligned}
& L_{0}^{2,1}=-h_{1} k_{2}+k_{4}-k_{1}^{2}-h_{1} h_{3}+h_{5}, \quad L_{0}^{3,2}=-k_{1} h_{2}+h_{2}^{2}-h_{5}+2 k_{4}-k_{1}^{2}, \\
& L_{0}^{3,1}=h_{1} k_{3}-2 h_{1} h_{4}+3 k_{5}+h_{2} h_{1}^{2}-2 k_{1} k_{2}-2 k_{1} h_{3}+h_{1}^{2} k_{1}-2 k_{2} h_{2}+h_{2} h_{3} .
\end{aligned}
$$

Уравнение спектральной кривой $\mathcal{C}: \operatorname{det}(\mu-L(\lambda))=0$ матришы Лакса (4.25) зависит от двух функций Казимира и четырех интегралов движения,

$$
\Phi(\mu, \lambda)=\mu^{3}-\mu\left(I_{1} \lambda+I_{2}\right)-\left(\lambda^{5}+I_{3} \lambda^{3}+I_{4} \lambda^{2}+I_{5} \lambda+I_{6}\right)=0 .
$$

Явные выражения для интегралов указаны в работе [25]. В методе Гельфанда-Захаревича [4] эта кривая возникает как функция Казимира для пуассонова пучка $P_{\lambda}=P_{1}-$ $\lambda P_{0}$, зависящего от параметра $\lambda[25]$. 
В исходном десятимерном пространстве данная интегрируемая система является квазиоднородной, так как соответствуюшее лагранжево многообразие представимо в виде

$$
\mathcal{C}^{(5)}: \quad \mathcal{C} \times \mathcal{C} \times \mathcal{C} \times \mathcal{C} \times \mathbb{R}^{2}
$$

В отличие от волчка Горячева-Чаплыгина обе функции Казимира $I_{1}$ и $I_{3}$ произвольны и линейны по переменным $k_{4}$ и $k_{5}$. Эти специфические особенности позволяют определить редукцию на поверхность уровня $S_{a b}$ функций Казимира $I_{1}=a$ и $I_{3}=b$ всех интегралов движения $\hat{I}_{j}$ [25]. Поверхность $S_{a b}-8$-мерный симплектический лист, на котором пуассонова структура становится невырожденной, а интегрируемая система - однородной. Соответствующее редуцированной системе четырехмерное лагранжево многообразие зависит от четырех значений интегралов движения $\alpha_{j}=\hat{I}_{j}$ и двух дополнительных параметров $a$ и $b$.

ПРЕДЛОЖЕНИЕ 8. Переменные разделения являются решениями системы уравнений

$$
\Phi(\mu, \lambda, \hat{I}, a, b)=0, \quad \frac{\partial}{\partial a} \Phi(\mu, \lambda)=0, \quad \frac{\partial}{\partial b} \Phi(\mu, \lambda)=0
$$

где функиия $\Phi$ определяет единственную плоскую кривую $\mathcal{C}(4.26)$, а $\hat{I}_{j}$ - ограничения интегралов $I_{j}$ на симплектический лист $S_{a b}$ общего положсения.

ДокАЗАТЕльство. Интегралы $\hat{I}_{j}$ в явном виде приведены в работе [25]. Подставляя эти интегралы в уравнения (4.27), можно доказать, что решения этих уравнений совпадают с собственными значениями тензора Нийенхейса. Эти же переменные совпадают с полюсами функции Бейкера-Ахиезера, нормированной на вектор $\vec{\alpha}=(1,0,0)$. Напомним, что при такой нормировке система уравнений (4.22), определяющая переменные разделения [5], имеет вид

$$
\begin{aligned}
L_{2,2} L_{3,3}-\mu\left(L_{2,2}+L_{3,3}\right)+\mu^{2}-L_{2,3} L_{3,2} & =0, \\
L_{1,2} L_{3,3}-L_{1,2} \mu-L_{1,3} L_{3,2} & =0, \\
L_{1,2} L_{2,3}-L_{1,3} L_{2,2}+L_{1,3} \mu & =0 .
\end{aligned}
$$

Здесь $L_{i, j}$ - элементы матрицы Лакса, зависящие от спектрального параметра $\lambda$.

Два последних уравнения системы (4.27) совпадают с двумя последними уравнениями системы (4.28). Если решить эти уравнения относительно переменной $\mu$ и подставить полученные решения в первые из уравнений систем (4.27) или (4.28), то наибольший обший делитель полученных уравнений будет совпадать с определителем тензора Нийенхейса. Отсюда следует, что решениями системы уравнений (4.27) являются переменные разделения.

С другой стороны, так же как для волчка Горячева-Чаплыгина, можно вместо редукции интегралов движения использовать для нахождения переменных разделения 
масштабное преобразование $\phi: S_{a b} \mapsto S_{c d}$, которое связывает симплектические листы с различными значениями операторов Казимира $I_{1}$ и $I_{3}$. Подобное преобразование можно определить, изменяя матрицу Лакса по правилу

$$
L \mapsto \tilde{L}(\lambda)=L(\lambda)+\left(\begin{array}{ccc}
0 & 0 & 0 \\
b_{1} I_{3}+b_{2} & 0 & 0 \\
a_{1} I_{1}+a_{2} & c_{1} I_{3}+c_{2} & 0
\end{array}\right), \quad a_{k}, b_{k} \in \mathbb{R} .
$$

Спектральная кривая новой матрицы сохраняет вид (4.26) после замены коэффициентов $I_{k} \mapsto \tilde{I}_{k}$, где

$$
\tilde{I}_{1}=\left(a_{1}+1\right) I_{1}+a_{2}, \quad \tilde{I}_{3}=\left(b_{1}+c_{1}+1\right) I_{3}+b_{2}+c_{2} .
$$

Выражения для остальных интегралов могут быть получены из определения матрищы Лакса.

Соответствующее лагранжево многообразие зависит от шести произвольных параметров. Однако параметры, отвечающие масштабному преобразованию и сдвигу операторов Казимира, порождают подобные уравнения,

$$
\frac{\partial}{\partial x_{1}} \Phi(\mu, \lambda)=0 \Leftrightarrow \frac{\partial}{\partial x_{2}} \Phi(\mu, \lambda)=0, \quad x_{k}=a_{k}, b_{k}, c_{k} .
$$

Таким образом, можно построить две различные системы уравнений вида $(2.2),(2.3)$

$$
\Phi(\mu, \lambda, \tilde{I}, a, b, c)=0, \quad \frac{\partial}{\partial a_{k}} \Phi(\mu, \lambda)=0, \quad \frac{\partial}{\partial b_{k}} \Phi(\mu, \lambda)=0
$$

и

$$
\Phi(\mu, \lambda, \tilde{I}, a, b, c)=0, \quad \frac{\partial}{\partial a_{k}} \Phi(\mu, \lambda)=0, \quad \frac{\partial}{\partial c_{k}} \Phi(\mu, \lambda)=0 .
$$

ПРЕДЛОЖЕНИЕ 9. Решения систем уравнений (4.29), (4.30) определяют два различных семейства переменных разделения, которые зависят от параметров $c_{1,2}$ или $b_{1,2}$.

Эти два семейства переменных связаны друг с другом каноническим преобразованием. Таким образом, мы доказали, что для данной системы можно построить переменные разделения, используя операторы Казимира. При этом необходимо либо определить ограничение всех интегралов движения на поверхность уровня $S_{a b}$ данных операторов, либо построить отображения, связывающие различные поверхности.

\section{5. ЗАКЛЮЧЕНИЕ}

В настоящее время сушествует два основных метода построения переменных разделения, использующих преобразование Беклунда [5], [16] или структуру ПуассонаНийенхейса [4], [6], [9], [25]. В одном из методов переменные разделения возникают как полюсы функции Бейкера-Ахиезера, в другом - как собственные значения тензора 
Нийенхейса. В обоих случаях исходный набор интегралов движения явно не используется при построении переменных разделения, а входит в доказательство разделимости построенных координат только на завершаюшей стадии.

Преобразования Беклунда сохраняют форму интегралов движения и связаны с автоморфизмами лиувиллева тора $\mathcal{T}^{n}$. Эти автоморфизмы действуют как композиции сдвигов $\varphi_{j} \mapsto \varphi_{j}+\Omega_{j}(s)$ угловых переменных [16], [26] и порождаются производными по касательным к лиувиллеву тору направлениям. В теории бигамильтоновых систем [4], [6], [25] используются только переменные действия $s_{j}(\alpha, a)$ и с их помошью определяются переменные разделения.

В данной работе предложен метод построения переменных разделения, в котором используются дополнительные параметры $a_{1}, \ldots, a_{m}$, входящие в определение переменных действия $s_{j}\left(I_{1}, \ldots, I_{n}, a\right)$. При этом для построения переменных разделения явно используются интегралы движения и производные по трансверсальным к лиувиллеву тору направлениям, так как уравнения (2.3) можно представить в виде

$$
\frac{\partial \Phi(\lambda, \mu)}{\partial a_{k}}=\sum_{j=1}^{n} \frac{\partial s_{j}}{\partial a_{k}} \frac{\partial \Phi(\lambda, \mu)}{\partial s_{j}}=0
$$

В силу этого данный метод можно рассматривать как альтернативньй к методу, основанному на преобразованиях Беклунда.

Основной проблемой в предлагаемом подходе является построение интегрируемых обобщений исходной системы, которые зависят от дополнительных параметров. Для решения этой проблемы можно использовать канонические преобразования для однородных интегрируемых систем или редукцию на поверхность уровня функций Казимира для квазиоднородных интегрируемых систем. В данной работе для ряда интегрируемых систем на орбитах коприсоединенного представления групा Ли была доказана возможность явного построения переменных разделения с использованием операторов Казимира, определяюших эти орбиты. Было бы интересно рассмотреть эту конструкцию переменных разделения в общей теории интегрируемых систем с фазовыми пространствами такого типа.

Благодарности. Работа выполнена при финансовой поддержке РФФИ (грант № 99-01-00698) и INTAS (грант № 99-01459).

\section{Список литературы}

[1] А. М. Виноградов, Б. А. Куперимидт. УМН. 1977. Т. 32. С. 175.

[2] В. И. Арнольд. Математические методы классической механики. М.: Наука, 1989.

[3] А. Г. Рейман, М. А. Семенов-Тянь-Шанский. Теоретико-групповые методы в теории интегрируемых систем. В сб.: Динамические системы-VII. Итоги науки и техники. Современные проблемы математики. Фундаментальные направления. Т. 16. Ред. Р. В. Гамкрелидзе. М.: ВИНИТИ, 1987. С. 119.

[4] I. M. Gel'fand, I. Zakharevich. On the local geometry of a bi-Hamiltonian structure. In: The Gelfand Mathematical Seminars 1990-1992. Eds. L. Corwin et al. Boston: Birkauser, 1993. P. 51. 
[5] E. K. Sklyanin. Progr. Theor. Phys. Suppl. 1995. V. 118. P. 35.

[6] I. M. Gelfand, I. Zakharevich. Webs, Lenard schemes and the local geometry of bihamiltonian Toda and Lax structures. math.DG/9903080; G. Falqui, F. Magri, M. Pedroni. Bihamiltonian geometry and separation of variables for Toda lattices. Ref. SISSA 139/1999/FM. Trieste: SISSA, 1999.

[7] M. Adler, P. van Moerbeke. Algebraic Completely Integrable Systems: a Systematic Approach. New York: Acad. Press, 1993.

[8] P. Vanhaecke. Integrable Systems in the Realm of Algebraic Geometry. Lect. Notes Math. V. 1638. New York: Springer, 1996.

[9] M. Blaszak. J. Nonlinear Math. Phys. 2000. V. 7. P. 213.

[10] P. Stäckel. Uber die Integration der Hamilton-Jacobischen Differential Gleichung Mittelst Separation der Variabel. Halle: Habilitationsschrift, 1891.

[11] A.P. Fordy. Physica D. 1991. V. 52. P. 204.

[12] E. G. Kalnins. Separation of Variables for Riemann Spaces of Constant Curvature. New York: Wiley, 1986.

[13] B. Kostant. Adv. Math. 1979. V. 34. P. 195.

[14] V. I. Inozemtsev. Commun. Math. Phys. 1989. V. 121. P. 629.

[15] V. B. Kuznetsov. J. Phys. A. 1997. V. 30. P. 2127.

[16] E. K. Sklyanin. Bäcklund transformations and Baxter's $Q$-operator. nlin.si/0009009.

[17] A. V. Tsiganov. J. Math. Phys. 1997. V. 38. P. 196.

[18] E. K. Sklyanin. The quantum Toda chain. In: Nonlinear Equations in Classical and Quantum Field Theory (Proc. Seminar Series Held at the Observatoire de Mendon, Mendon, and at the Univ. Pierre and Marie Curie, Paris, Oct. 1983 - Oct. 1984). Lect. Notes Phys. V. 226. Ed. N. Sanchez. Berlin: Springer, 1985. P. 196.

[19] Y. Zeng. J. Phys. A. 2000. V. 33. P. 621.

[20] А. В. Цызанов. ТМФ. 1998. Т. 115. С. 3.

[21] А. В. Цыганов. ТМФ. 1999. Т. 120. С. 27.

[22] C. Bechlivanidis, P. van Moerbeke. Commun. Math. Phys. 1987. V. 110. P. 317.

[23] E. K. Sklyanin. J. Sov. Math. 1985. V. 31. P. 3417.

[24] A. I. Bobenko, V. Kuznetsov. J. Phys. A. 1988. V. 21. P. 1999.

[25] Г. Фалъки, Ф. Магри, Г. Тондо. ТМФ. 2000. Т. 122. С. 219.

[26] А. П. Веселов. УМН. 1991. Т. 46. № 5. С. 3. 